\title{
First look at HRCAM images from Dome A, Antarctica
}

\author{
Geoff Sims ${ }^{1}$, Michael C. B. Ashley ${ }^{1}$, Xiangqun Cui ${ }^{2}$, \\ LongLong Feng ${ }^{3,4}$, Xuefei Gong ${ }^{2,4}$, Zhongwen $\mathbf{H u}^{2,4}$, \\ Jon S. Lawrence ${ }^{5,6}$, Daniel M. Luong-Van ${ }^{1}$, Zhaohui Shang ${ }^{4,7}$, \\ John W. V. Storey ${ }^{1}$, Nick Tothill ${ }^{8}$, Lifan Wang ${ }^{3,4,9}$, Huigen Yang ${ }^{4,10}$, \\ Ji Yang ${ }^{3}$, Xu Zhou ${ }^{4,11}$ and Zhenxi Zhu ${ }^{3,4}$ \\ ${ }^{1}$ School of Physics, University of New South Wales, Sydney NSW 2052, Australia \\ email: g.sims@unsw.edu.au \\ ${ }^{2}$ Nanjing Institude of Astronomical Optics \& Technology, Nanjing 210042, China \\ ${ }^{3}$ Purple Mountain Observatory, Nanjing 210008, China \\ ${ }^{4}$ Chinese Center for Antarctic Astronomy, China \\ ${ }^{5}$ Department of Physics and Astronomy, Macquarie University, Sydney NSW 2109, Australia \\ ${ }^{6}$ Australian Astronomical Observatory, Sydney NSW 1710, Australia \\ ${ }^{7}$ Tianjin Normal University, Tianjin 300074, China \\ ${ }^{8}$ University of Western Sydney, Sydney NSW, Australia \\ ${ }^{9}$ Department of Physics and Astronomy, Texas A\&M University, College Station 77843, USA \\ ${ }^{10}$ Polar Research Institute of China, Shanghai 200136, China \\ ${ }^{11}$ National Astronomical Observatories, Chinese Academy of Science, Beijing 100012, China
}

\begin{abstract}
HRCAM (High Resolution CAMera) is a Canon 50D 15-megapixel digital SLR camera equipped with a Sigma $4.5 \mathrm{~mm} \mathrm{f} / 2.8$ fish-eye lens. It was installed at Dome A on the Antarctic plateau in January 2010 and photographs the sky every 15 minutes. Primarily functioning as a site-testing instrument, data obtained from HRCAM provide valuable statistics on cloud cover, sky transparency and the distribution and frequency of auroral activity. We present a first look at data from HRCAM during 2010, including an overview of how we intend to reduce the images. We also demonstrate the potential of stellar photometry by using linear combinations of the in-built Canon RGB filters to convert instrumental magnitudes into the photometric BVR bands.
\end{abstract}

Keywords. Dome A, site testing, aurora, sky brightness, sky transparency

\section{Introduction}

The highest point on the Antarctic plateau, Dome A, has been home to a host of site testing and scientific instruments since the first Plateau Observatory (PLATO) was installed in 2008. HRCAM (High Resolution CAMera) is one such instrument, whose purpose is twofold: primarily it is a site testing camera to provide statistics on cloud cover, auroral distribution and sky transparency; secondly it serves as a visual reference to check any epochs at which other instruments record unusual results. In the case of HRCAM-3 at Ridge A (see Section 2), in the absence of a meteorological tower it was able to provide an indication of wind speed and direction by the direct observation of a flag. In many ways HRCAM is complementary to various instruments such as Nigel (Sims et al. 2010), Gattini (Moore et al. 2010) and CSTAR (Yuan et al. 2008), since it monitors similar phenomena but with the added advantage of all-sky coverage. 


\section{Instrumentation \& Control}

Instrumentation: HRCAM is composed of a Canon 50D digital SLR (DSLR) camera and a Sigma $4.5 \mathrm{~mm} \mathrm{f} / 2.8$ fish-eye lens. This combination provides full sky coverage on the camera's 15.1 megapixel and $1.6 \mathrm{x}$ crop $\dagger$ sensor. The camera and lens assembly are contained in an enclosure which is designed to operate down to $-80^{\circ} \mathrm{C}$. The lens is flush with the top and exposed directly to the sky. A reflective metal cover surrounds the lens and is designed to shed snow; it has a low emissivity so that it can be heated efficiently. Figure 1 shows a photograph of HRCAM, shortly after being installed at Dome A. The holes on the bottom right in the Figure are for a dehumidifying system. The shutter is rated for 100,000 actuations, limiting the cadence of observations (based on the anticipated duration of the experiment).

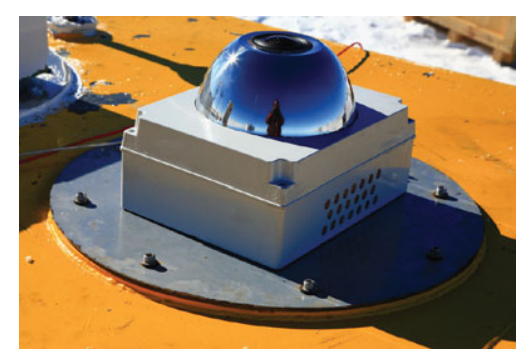

Figure 1. HRCAM installed on the roof of the PLATO instrument module at Dome A in 2010.

Control: A single Milspec connector provides 24 VDC power and a 100 Mbps LAN connection. The enclosure includes an ARM-based computer running Linux, and the camera is controlled via a USB connection using gphoto2. The raw images are stored on an array of four $500 \mathrm{~GB}$ spinning hard-disks inside PLATO. Exposures are taken every 15 minutes and vary from $1 / 2000$ th of a second to 120 seconds depending on the sky brightness, and are automatically set by the software. Images are shot in 14-bit raw (.CR2) format, and thumbnail representations $(80 \times 80 \mathrm{px}$ JPEG images; $\sim 5 \mathrm{kB}$ in size) are sent back via PLATO's Iridium satellite link. Despite the low resolution of the thumbnails, they are large enough to visually detect cloud, "diamond dust" (including $22^{\circ}$ halos) and aurorae.

HRCAM clones: In addition to the original HRCAM at Dome A (which ran throughout 2010), there are two other exact clones on other sites on the high plateau: HRCAM-F at Dome F (2011), and HRCAM-3 at Ridge A (2012). As a result of the various deployment dates, there is so far virtually no overlap in data between any of the various HRCAMs.

\section{Reduction}

As with most consumer DSLRs, the Canon 50D uses a Bayer colour filter array (CFA). The structure of this array is different from conventional astronomical CCD imagers and thus requires a different reduction process. We first convert the raw images into FITS files using dcraw (Coffin 2012) and cr2fits.py (Jordahl 2012). The 14-bit data are preserved with no scaling or white balance adjustments. The resulting image is then split into four colour channels: $\mathrm{R}, \mathrm{G}_{1}, \mathrm{G}_{2}$ and $\mathrm{B}$, each channel being $25 \%$ of the original resolution. A schematic of the pipeline is shown in Figure 2.

$\dagger$ Most consumer-grade digital cameras, including the Canon 50D, have CCD sensors smaller than the standard $36 \times 24 \mathrm{~mm}(35 \mathrm{~mm})$ film format, which results in the sensor capturing only a subset of the 'full frame' image. 


\section{RAW (.CR2) IMAGE $\underset{\text { DCRAW; CR2FITS.PY }}{\longrightarrow}$ FITS FILE}

(ZOOMED REGION SHOWS BAYER FILTER STRUCTURE)
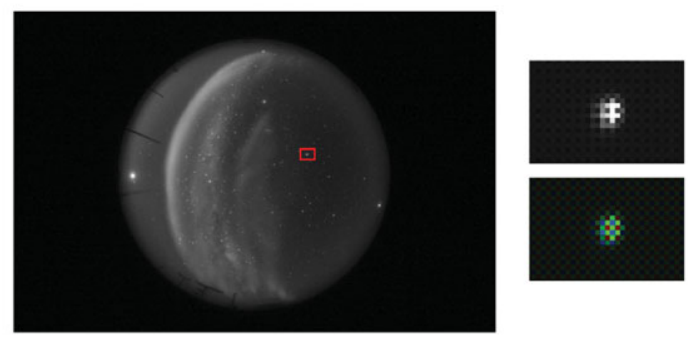

\section{SPLit AND COLLAPSE THE 4 COLOUR CHANNELS}

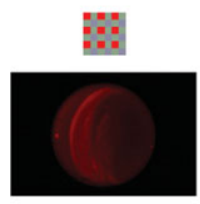

RED

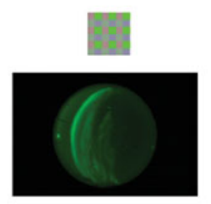

GREEN 1

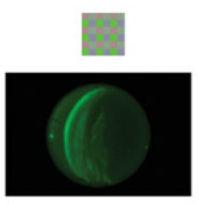

GREen 2

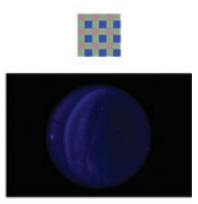

BLUE

Figure 2. Data reduction pipeline.

\section{Results \& sample images}

Photometry: As shown in Figure 3, the transmission profiles of the Bayer CFA are different to the standard BVR photometric filters. We investigated the possibility of using linear combinations of the colours to more closely match the standard filters. Aperture photometry was performed on a sample green image, using 14 stars, each at a similar airmass and with $0<m_{v}<6$. The rms error associated with the simple photometry was 0.29 mag. With a colour correction term $(B-G)$ added (determined using a least-squares method), this error dropped to $0.23 \mathrm{mag}$; an improvement of $\sim 20 \%$.
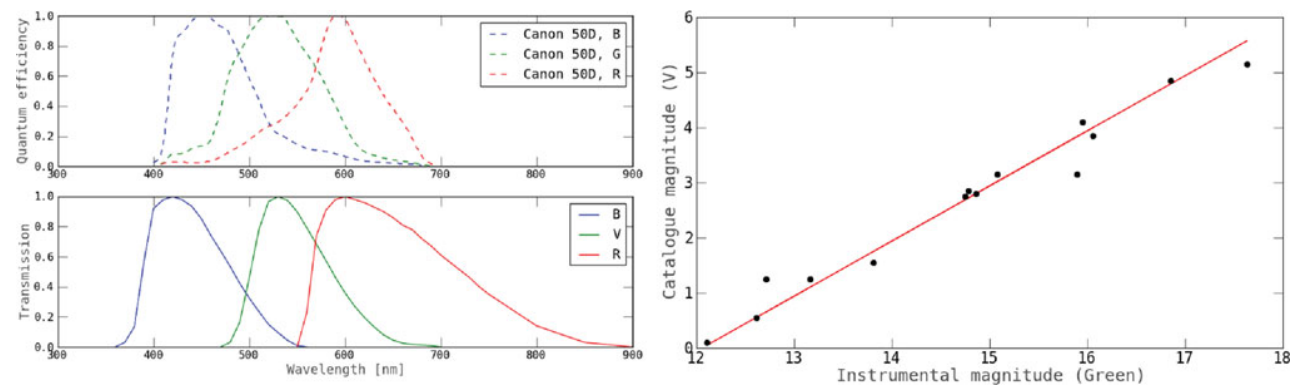

Figure 3. Left: comparison of Canon RGB and photometric BVR filter profiles; Right: Preliminary photometry test.

Sample images: Four sample images are given in Figure 4.

\section{Acknowledgements}

The author acknowledges the Astronomical Society of Australia (ASA) for providing travel support to attend this meeting. 

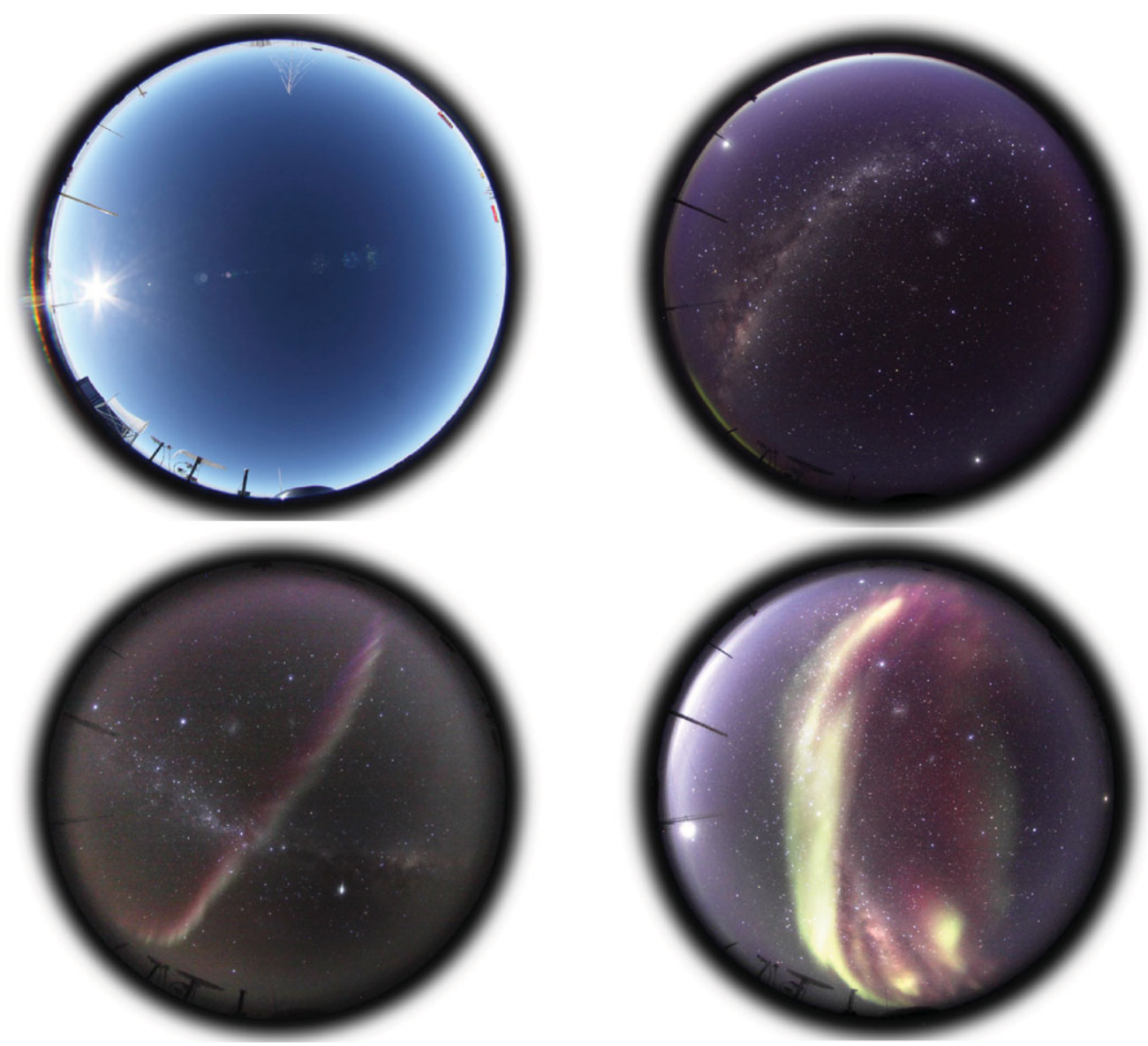

Figure 4. Sample HRCAM images. Upper left: blue sky during summer; Upper right: clear sky during winter, green aurorae visible on the horizon; Lower left: auroral arc with bright meteor; Lower right: bright auroral display.

\section{References}

Coffin, D. 2012, $W W W$, http://www.cybercom.net/ dcoffin/dcraw/

Jordahl, K. 2012, $W W W$, https://github.com/kjordahl/cr2fits

Moore, A. et al. 2010, Proc. SPIE, 7733, 77331S

Sims, G. et al. 2010, Proc. SPIE, 7733, 77334M

Yuan, X. et al. 2008, Proc. SPIE,7012, 70124G 